\title{
Neuroplasticidad asociada a miembro fantasma
}

\author{
R. de la Puerta Huertas \\ Enfermería. Hospital Universitario Marqués de Valdecilla (HUMV). Santander, Cantabria
}

De la Puerta Huertas R. Neuroplasticidad asociada a miembro fantasma. Rev Soc Esp Dolor 2014; 21(6): 345-350.

\begin{abstract}
In recent decades, the concept of neuroplasticity has taken clear relevance associated with the patient's health and learning and behavior in the healthy individual. This ability of the nervous system involves assimilation, reorganization and modification of our biological mechanism, biochemical and physiological.

With the advent of new scientific technologies that have appeared in recent years, we respond and maintain this concept of plasticity of the nervous system as a basic premise of being susceptible to external changes and dynamic. The phantom limb experience exposes permanently, the existence of an underlying mental body map and modifies the experience with our body, whose nervous system has the ability of cortical reorganization by sensory stimulation, sensory, endocrine and motor. The relationship between phantom and neuroplasticity is complex, difficult to investigate despite scientific breakthroughs, yet is dynamic, and that this capacity meets the needs of human health at different stages of his life.
\end{abstract}

Key words: Neuroplasticity. Pain. Phantom limb. Neuromodulation.

\section{RESUMEN}

En las últimas décadas, el concepto de neuroplasticidad ha tomado clara relevancia asociado a la salud del paciente y al aprendizaje y conducta en el individuo sano. Esta capacidad del sistema nervioso implica asimilación, reorganización y modificación de nuestro mecanismo biológico, bioquímico y fisiológico.

Con el advenimiento de nuevas tecnologías científicas que han aparecido en los últimos años, respondemos y mantenemos a este concepto de plasticidad del sistema nervioso como premisa base de ser susceptible a cambios externos y dinámicos. La experiencia del miembro fantasma pone al descubierto, de forma permanente, la existencia de un mapa corporal mental que subyace y modifica la experiencia con nuestro cuerpo, cuyo sistema nervioso posee la capacidad de reorganización cortical por estimulación sensitiva, sensorial, endocrina y motora. La relación entre miembro fantasma y neuroplasticidad es compleja, difícil de investigar a pesar de los avances científicos obtenidos y, a la vez, es dinámica, ya que esta capacidad responde a las necesidades de salud en el ser humano en las diferentes etapas de su vida.

Palabras clave: Neuroplasticidad. Dolor. Miembro fantasma. Neuromodulación.

\section{INTRODUCCIÓN}

Plasticidad funcional y estructural son propiedades bien conocidas del sistema nervioso que se producen después de lesiones drásticas, tales como la pérdida de parte del cuerpo.

Cuando una persona está aprendiendo una nueva habilidad, recuperándose de una lesión o participa en una programación de intervención, tienen lugar cambios plásticos a nivel central. Sin embargo, esa neuroplasticidad que sucede en el cerebro, intensamente estudiada en animales, no es fácilmente accesible en los seres humanos a los que los métodos invasivos de investigación no se pueden aplicar sin válidas razones terapéuticas.

Esto nos lleva a una difícil búsqueda de información. Los modelos de animales a su vez no proporcionan información acerca de las funciones mentales superiores como el lenguaje o la música (1). 
Estudios previos han indicado que la amputación o desaferenciación de una extremidad induce cambios funcionales en la zona cortical S1 (localización y discriminación de sensación y dolor) y M1 (corteza motora) relacionadas con el dolor del MF. Varios resultados demuestran una reasignación funcional de la zona $\mathrm{S} 1$ en amputados de miembro inferior. Sin embargo, en contraste con estudios anteriores, los cambios neuroplásticos no parecen ser dependientes rigurosamente del dolor de miembro fantasma, ya que estos cambios también se producen en los sujetos que informaron de la sensación del MF sin dolor (2).

Nuevas investigaciones sobre estos cambios corticales han llevado a una reevaluación de las patologías que cursan con dolor crónico y sus tratamientos. En el caso del síndrome del MF con dolor (SMFD) se replantean como una disfunción a nivel central del sistema nervioso (3).

La neuroplasticidad se produce en una variedad de niveles. Décadas de investigación han demostrado que produce cambios sustanciales en las áreas más bajas del procesamiento neocortical y que estos cambios pueden alterar profundamente el patrón de activación neuronal en respuesta a la experiencia (4).

Los cambios producidos por la plasticidad neuronal vienen a desembocar en:

- Activación de nuevas regiones cerebrales (re-wiring o re-routing).

- Re-mapeo del SNC:

- Con cambios en la representación neural que pueden ocurrir como respuesta a la demanda del medio.

- La extensión del área de respuesta ha sido demostrada en la corteza somatosensorial humana.

- Los cambios dinámicos pueden cristalizarse en cambios duraderos inducidos por la plasticidad inducida por el aprendizaje (5).

\section{ESQUEMA CORPORAL}

El esquema corporal suele identificarse con el concepto de imagen corporal, pero a veces se realiza una distinción entre ambos términos, refiriéndonos con "imagen corporal" a la representación consciente y con "esquema corporal" a la representación inconsciente.

En ocasiones se identifica "imagen corporal" con la representación conceptual y verbalizable de nuestro propio cuerpo. Finalmente, no podemos ignorar que la "imagen corporal" está impregnada de connotación cultural y sujeta a mutaciones en relación con los usos de cada época. De todas formas, estos términos se han usado de forma indistinta.

La representación de nuestro propio cuerpo, necesaria para nuestra interacción con el entorno, está basada en tres sistemas interdependientes (6):

1. La conciencia de nuestro cuerpo a través de las aferencias táctiles y sentidos, aferencias vestibulares, cines- tésicas y visuales nos proporcionan una información que integrada y procesada, aporta un conocimiento sobre la configuración y posición corporal.

Es más, en ausencia de estas aferencias poseemos un "sentimiento" de la posición de las partes del cuerpo (podemos señalar la punta de nuestra nariz sin vacilar). Este conocimiento básico de los límites y la disposición de nuestro propio cuerpo es lo que se denomina "esquema corporal" (7).

2. El conocimiento general de nuestro cuerpo y sus partes. Este conocimiento puede ser de diferentes categorías:

- Un conocimiento lexical y semántico que define los nombres, categorías y función de cada una de las partes del cuerpo.

- Un conocimiento topográfico de la distribución espacial de las partes del cuerpo. Este conocimiento proporciona información sobre la posición de cada parte concreta del cuerpo, la relación de proximidad que existe entre ellas y los límites de cada una (7).

3. La información que poseemos sobre la actual situación y configuración de nuestro cuerpo (espacio y dirección corporal) es la referencia necesaria para planear y ejecutar movimientos dirigidos hacia objetivos externos (espacio de apresamiento y espacio de acción) (8).

Una alteración en la propia percepción de nuestro esquema corporal podría dar lugar a una experiencia de miembro fantasma o a una heminegligencia personal.

\section{NEUROPLASTICIDAD}

La neuroplasticidad se considera como la capacidad que tiene el tejido neuronal de reorganizar, asimilar y modificar los mecanismos biológicos, bioquímicos y fisiológicos implicados en la comunicación intercelular, para adaptarse tras los estímulos recibidos.

Esta característica implica modificaciones del tejido neural que incluye la regeneración axonal, la colaterización, la neurogénesis, la sinaptogénesis y la reorganización funcional (9).

Este proceso pone al descubierto la susceptibilidad a los cambios fisiológicos del sistema nervioso. Esos cambios son un proceso dinámico que dura toda la vida (4).

\section{FENOMENOLOGÍA DEL MIEMBRO FANTASMA}

La descripción del MF ya fue realizada hace siglos por Paré, Descartes y Von Haller. Los estudios sistemáticos más antiguos sobre este trastorno fueron realizados 
por Gueniot en 1861, Weir Mitchell en 1872, Charcot en 1892, Abbatucci en 1894, Pitres en 1897, Head y Holmes en 1911 y Pick en 1915.

La experiencia de miembro fantasma constituye un claro argumento a favor de la existencia de un esquema corporal mental que subyace y modifica la experiencia con nuestro propio cuerpo (10).

Las experiencias de MF no solamente ocurren tras la pérdida de extremidades, sino también tras la pérdida de otros órganos como los ojos, los dientes, los genitales externos o las mamas. Sin embargo, la resección de un órgano interno no produce el fenómeno de MF.

Después de una amputación, es frecuente que hasta un $90 \%$ de los sujetos sigan percibiendo el miembro perdido. Pero habitualmente, con el paso del tiempo, esta sensación va desapareciendo. Cuando persiste, reaparece intermitente bajo ciertas condiciones. Ocurre menos en casos de retraso mental y situaciones de estrés.

En la descripción del MF es importante distinguir dos grupos de alteraciones:

1. En primer lugar, la experiencia del MF como una percepción del miembro amputado en lo que se refiere a sus características espaciales. En este sentido constituiría la persistencia del esquema mental corporal.

2. En segundo lugar, a diferencia de lo anterior, estarían las sensaciones percibidas (tálamo) procedentes del MF (parestesias, dolor, sensación de pesadez, de calor, frío, calambres, etc.).

Esta distinción entre el fenómeno de miembro fantasma en sí mismo y las sensaciones percibidas en el miembro es esencial, porque su patogénesis es diversa, y porque son experimentados por el sujeto de forma distinta.

El fenómeno de MF constituye una parte de la experiencia integral normal de la propia corporalidad.

Stetter establece la siguiente distinción (11): "Las sensaciones fantasmas tienen características de sensaciones, mientras que la experiencia fantasma es una experiencia de totalidad".

El miembro está constituido por componentes somestésicos. Toda información somestésica referente a apreciación del tamaño, longitud, peso, posición y movimiento está presente.

Las parestesias son las más frecuentes. Ocurren de forma inmediata tras la amputación y son más agudas en las partes distales.

\section{Miembro fantasma sin amputación}

La percepción de un miembro fantasma puede ocurrir no solamente tras una amputación sino también cuando las vías sensitivas aferentes de ese miembro se dañan, como es el caso de las neuropatías periféricas, plexopatías, lesiones medulares y lesiones cerebrales subcorticales.
En estos casos, el miembro desaferentizado puede experimentarse como un miembro adicional. La sensación de MF también puede ocurrir como experiencia transitoria de origen epiléptico. La experiencia de MF adicional o tercer miembro es a veces incapacitante y permanente, sobre todo en las lesiones medulares.

En estos enfermos, la experiencia del MF puede asociarse con sensaciones de micción, defecación o parestesias. Los MF tras lesiones medulares pueden no estar sujetos a la experiencia telescópica (descrito por Gueniot en 1861: "El MF se hace gradualmente más pequeño y peor definido, y la porción distal que permanece estable se aproxima finalmente al muñón hasta desaparecer dentro del mismo") (11).

\section{BASES NEUROANATÓMICAS}

Tras la amputación de un miembro o la pérdida de sus vías aferentes, se produce una remodificación de la representación topográfica a nivel parietal, en la corteza sensorial primaria (S1) y se realiza una traslación desde la zona dedicada al miembro amputado hasta una zona adyacente del Homúnculo de Penfield (12).

El corte de las aferencias nerviosas que causa la amputación de una extremidad produce una serie de cambios anatómicos y fisiológicos que afectan no sólo a los axones de las neuronas sensoriales primarias, sino también a partes alejadas de estas, como los cuerpos celulares y las sinapsis en la espina dorsal.

Es conocido que la transmisión nerviosa de las sensaciones somáticas se produce mentalmente por medio de las fibras A-beta, A-delta y C. Las A-beta se tratan de fibras mielinizadas asociadas con mecanorreceptores altamente especializados de la periferia. Las A-delta y $\mathrm{C}$ responden ante estímulos de alta intensidad y su activación genera una sensación dolorosa. Se ha hipotetizado que en un estado de dolor crónico se produce probablemente un cambio en la excitabilidad de las fibras A-beta, originando dolor ante estímulos de baja intensidad (Treede, 1992; Wolf y Doubelll, 1994) (13). Podría considerarse como un error en el proceso de plasticidad neuronal, representacional de la corteza cerebral. Sin embargo, la experiencia del MF puede persistir incluso cuando no hay una estimulación táctil de las zonas cutáneas adyacentes (donde se hubiera podido trasladar la representación topográfica del miembro amputado), por lo que parece que el sustrato neurológico de esta alteración no debe estar en la misma corteza sensorial primaria.

Se han descrito algunos enfermos que tras una lesión del lóbulo parietal posterior con preservación de la corteza sensorial primaria, han perdido la percepción del MF contralateral. Se diría pues, que la integridad de áreas de asociación en el lóbulo parietal posterior contralateral es necesaria para la existencia del trastorno. 
Rev. Soc. Esp. del Dolor, Vol. 21, N. ${ }^{\circ}$ 6, Noviembre-Diciembre 2014

No se ha descrito ningún caso en el que el sujeto tenga esta alteración cuando existe un daño localizado exclusivamente en la corteza sensorial primaria; parece obvio que la preservación de esta área es también necesaria para la percepción del MF.

Es necesaria, aunque no suficiente, la preservación de una corteza sensorial primaria, en la que se ha producido un error en el proceso de plasticidad topográfica tras la pérdida o desaferentización de un miembro. La remodificación en la representación de las diferentes partes corporales se realiza de forma errónea.

En vez de suprimirse las áreas donde está representado corticalmente el miembro amputado, estas se siguen conservando o simplemente se trasladan, lo que da lugar a una interpretación errónea de esa información. Esta interpretación se lleva a cabo en áreas de asociación más posteriores, en el lóbulo parietal donde residiría el supuesto esquema corporal mental.

Estudios demuestran que la representación homuncular de la superficie corporal en la corteza somatosensorial está sujeta a modificaciones funcionales cuando se cortan las aferencias nerviosas que transmiten la información somatosensorial desde la periferia. Estas alteraciones afectan sólo a los mapas corticales sobre el hemisferio encargado de procesar información somatosensorial procedente de la mitad corporal correspondiente a la amputación.

La percepción de un miembro fantasma demuestra la existencia de un esquema corporal mental que persiste incluso tras perder su correlato real. Una vez demostrada su existencia, nos podríamos plantear si este esquema corporal mental es innato o adquirido (12).

A favor de que sea innato tenemos la experiencia del MF en niños pequeños con una ausencia congénita de miembros. Sin embargo, la frecuencia de esta alteración de la percepción corporal es mucho menor cuanto menor es la edad de los sujetos que pierden su miembro. Esto sugiere que la percepción del esquema corporal se va haciendo más intensa y duradera con la experiencia continuada de la propia corporalidad.

Las niñas no tienen experiencia de "mamas fantasma" antes de que estas se desarrollen, lo que va también en contra de la existencia de un esquema corporal innato. Algo similar sugiere el hecho de que la experiencia del MF va desapareciendo conforme transcurre el tiempo tras la amputación de un miembro (6).

Cabe resaltar que la plasticidad neural permite cambios de adaptación y/o reorganización en condiciones normales o patológicas.

Es posible inducir neuroplasticidad en los humanos por medio de desaferentación central. Dichas intervenciones han permitido avanzar en la comprensión de la plasticidad en individuos normales y aún en condiciones patológicas, al estudiar sus mecanismos de generación en áreas motoras, sensitivas y sensoriales.

\section{NEUROMODULACIÓN}

En términos estrictos, el concepto de neuromodulación se refiere a la capacidad de las neuronas de alterar las propiedades eléctricas en respuesta a los cambios bioquímicos, resultado de la estimulación hormonal o sináptica (14).

En los últimos años se ha manifestado de forma clara que el dolor incluye características periféricas y centrales. Los científicos actualmente poseen buena evidencia de los cambios a nivel cortical que se producen cuando los pacientes sufren dolor crónico (3).

El concepto moderno de la neuromodulación emergió hacia 1970, cuando Greenfield mencionó que este proceso es "una respuesta variable a un estímulo invariable", asociando esta a procesos eléctricos de excitación neuronal (15).

Con el paso del tiempo, otros autores mencionaron este proceso no sólo involucrado en el potencial de acción sino que incluía el intercambio de sustancias que ejercen, al parecer, efectos prolongados sobre la membrana y la excitabilidad neuronal.

La neuromodulación se ha caracterizado por la aplicación de electroterapia, y de una forma más reciente se ha incluido la estimulación magnética (EM) a altas y bajas densidades, la estimulación somatosensorial y somatoperceptual, la neuronavegación electromagnética, e inclusive la acupuntura.

La neuromodulación difiere del concepto clásico de la transmisión nerviosa. En este último caso la información recibida utiliza diversos neurotransmisores que van desde la región presináptica a la postsináptica, haciendo que esta última se despolarice o hiperpolarice produciendo un efecto inmediato y que puede durar hasta cientos de milisegundos. Cabe aclarar que en la neuromodulación de la región postsináptica no depende tanto del neurotransmisor cuanto más del receptor al que se liga, denominado receptor metabotrópico (16). En este sentido mientras el receptor inotrópico clásico afecta la permeabilidad de manera directa, el metabotrópico produce cambios en la neurona postsináptica por medio de la activación intracelular molecular, utilizando los "segundos mensajeros". Y aunque los efectos del primer mensajero se inactivan rápidamente, los de segundo mensajero pueden durar varios días. Más aún, algunas proteínas pueden afectar al genoma de una célula postsináptica, alterando de manera permanente su actividad (16).

\section{REORGANIZACIÓN CORTICAL EN MF}

Diferentes hipótesis han sido propuestas, entre ellas la de Melzack (1999), por la cual una red innata produce un patrón único de actividad neural que representa el esquema corporal.

Cuando este "cableado" no puede actualizar su información, permanece sin cambios, incluso después de una ampu- 
tación, genera los conflictos de percepción a que se refiere en los casos del síndrome del MF (Doetsch 1997-Sirigu 2008).

Se ha combinado la resonancia magnética funcional (fRM) y tensor de difusión (DTI) para investigar la plasticidad cortical y del cuerpo calloso en los amputados de miembros inferiores con "dolor" o sensaciones fantasmas, para aclarar si los fenómenos de plasticidad se pueden generalizar más allá de la amputación del miembro superior con el dolor fantasma y para probar si podían ser relacionados con enfermedades del cuerpo calloso.

Se encontró que el hemisferio desaferenciado muestra una superposición, la representación ampliada del muñón (después de la estimulación del muñón) y de la extremidad intacta (tras estimulación). Estos cambios estructurales se producen en sectores somatomotriz del cuerpo calloso.

La RM convencional no mostró anormalidades en el cerebro de los amputados. Por el contrario, fRM y DTI revelaron claros cambios en la sustancia blanca sobre funciones y microestructurales en amputados. Ninguno de los pacientes reportó dolor durante la estimulación táctil en exploración previa o entrevista.

En los amputados la estimulación sensorial del muñón resultó en la activación de S1 y M1 contralateral a la amputación (hemisferio desaferenciado), además de la zona del motor suplementario (M2). Otros sitios de activación significativos fueron ínsula, S2, el tálamo y el cuerpo estriado del hemisferio contralateral a la amputación.

De acuerdo con resultados de experimentos realizados en amputados de extremidades superiores se mostró una ampliación de la representación del muñón en la corteza desaferenciada, además de una reorganización funcional en el hemisferio "no desaferenciado". También encontraron deformidades estructurales de los surcos centrales en algunos pacientes y del lóbulo parietal contralateral atrófico, lo que sugiere una reorganización funcional bilateral y anatómica (2).

En muchos individuos, los fenómenos del MF aparecen tan pronto como 24 horas después de la amputación traumática, y pueden estar relacionados con el desenmascaramiento o la reactivación de conexiones pre-existentes, conduciendo a la supresión de la inhibición local (Borsook 1998; Ramanchadrán y Hirstein 1998; Werhahn 2002; Giummarra 2008).

Por otra parte, en algunos casos tras amputaciones, eran necesarios semanas o meses para la aparición de sensaciones fantasma, incluyendo el dolor (Pascual Leone, 1996).

En la reorganización somatosensorial cerebral funcional y estructural se necesitan estudios futuros que comparen directamente los casos de "dolor" y "no dolor" para explorar de forma exacta si existe dolor o no en el MF.

Ramachandrán y Hirstein (1998) propusieron un modelo multifactorial para explicar las sensaciones fantasmas que depende de la integración de experiencias de reasignación y vívidos recuerdos somáticos de la extremidad ori- ginal y de la imagen interna genéticamente determinada del cuerpo.

Además, las referencias cruzadas entre la relación de MF y miembro intacto ha ganado recientemente importancia (2).

\section{Dolor en el miembro fantasma}

El dolor del MF forma parte de los síndromes de dolor crónico difíciles de tratar. Las opciones para el tratamiento del MF son en gran parte implicadas por el nivel de comprensión de los mecanismos y la naturaleza del MF. Resultados clínicos y de investigación reconocen el carácter neuropático del MF y sugieren que ambos mecanismos, tanto el periférico como el central, incluidos los cambios de plasticidad en el SNC pueden contribuir al MF.

Los estudios de neuroimagen en MF han indicado una relación clara entre MF y los cambios neuroplásticos. Además, se ha demostrado que los cambios patológicos neuroplásticos podrían ser revertidos, y existe una relación entre una mejora (reversión) de los cambios neuroplásticos en el MF y el alivio del dolor.

Estos hallazgos facilitaron la exploración de nuevas estrategias de tratamientos neuromoduladores, sumándose a la variedad de enfoques de tratamientos en el MF.

En general las opciones de tratamientos disponibles para MF incluyen: el tratamiento farmacológico, las estrategias no invasivas no farmacológicas de apoyo (p. ej., neuromodulación mediante estimulación magnética transcraneal, terapia de retroalimentación visual, estimulación eléctrica transcutánea de nervio periférico, fisioterapia, reflexología o varios enfoques psicoterapéuticos).

La capacidad de inducir plasticidad cortical con técnicas no invasivas de estimulación cerebral ha brindado oportunidades nuevas y emocionantes para examinar el papel de la corteza cerebral humana durante una variedad de comportamientos. Además, de manera importante, la inducción de cambios duraderos en la excitabilidad cortical puede, bajo ciertas condiciones, de forma reversible, modificar la conducta y comportamiento e interactuar con el aprendizaje normal.

En los últimos avances se ha demostrado que una multitud de factores determinantes pueden influir en la magnitud y dirección de la plasticidad inducida en el cerebro humano. Estos factores juegan un papel importante en la explicación de la conocida variabilidad de la respuesta individual. Es importante tener en cuenta que un número de estos factores como: perfil genético, edad, hora del día (por su relación con el cortisol), sexo, influencias farmacológicas, ejercicio-actividad, motivación, etc., puede interactuar entre sí dando como resultado una influencia compleja multifactorial en la inducción de la neuroplasticidad en un caso terapéutico, como en uno sano. Teniendo en cuenta todos estos factores determinantes 
mencionados anteriormente, se predecirá mejor la respuesta de plasticidad individual en cada caso para mejorar la eficacia con un propósito terapéutico (17).

\section{NEURO-REHABILITACIÓN. CONCLUSIONES}

La rehabilitación es el mejor método conocido para facilitar la expresión de la plasticidad neural. Los orígenes científicos de la rehabilitación neurológica son relativamente recientes. El enfoque farmacológico usado por todas las disciplinas biomédicas ha nublado, y en ocasiones eliminado, de la mente de muchos profesionales de la salud, la posibilidad de ofrecerles un programa de rehabilitación neurológica con orientación científica (18).

Deben desarrollarse terapias que dirijan su objetivo principal a modificar la alteración de los mapas corticales que se representan en los pacientes amputados.

Actualmente esto es posible debido a los avances científicos hechos por diferentes grupos (19), incluyendo quienes han demostrado el uso de nuevas herramientas, como la estimulación magnética (EMT) (20) de alta y baja intensidad o las corrientes directas, la imaginería motora graduada (IMG) a través de las neuronas espejo (NE) (3) en pacientes con MF, entre otros avances científicos con los que se permite demostrar que el sistema nervioso adulto puede experimentar reorganización de la corteza somatosensorial aun en épocas tardías, algo considerado hasta hace poco como un imposible fisiológico.

Es importante que la neuro-rehabilitación sea holística pero individualizada, inclusiva y participativa; debe generar independencia, aplicarse de por vida si fuese necesario; siempre oportuna de acuerdo con las necesidades del paciente y orientada hacia la comunidad.

Esto implica un abordaje interdisciplinario, llevado a cabo por un equipo con experiencia en el área, integrado por profesionales con diferentes formaciones y enfoques científicos, y liderado necesariamente por un especialista en neuro-rehabilitación (21).

Los elementos teóricos y prácticos ayudan a establecer un adecuado control sensori-motor de los pacientes, con el fin de potenciar la recuperación funcional y la mejora de la calidad de vida en estos individuos, que es el objetivo principal y el aval de un avance científico bien encaminado hacia una ciencia con futuro.

\section{CORRESPONDENCIA:}

Rosmari de la Puerta Huertas

Servicio de Enfermería

Hospital Universitario Marqués de Valdecilla

Avda. Valdecilla, s/n

39008 Santander

e-mail: rosmariph@yahoo.es

\section{BIBLIOGRAFÍA}

1. KujalaT, Näätänen R. La adaptación del cerebro: una perspectiva neurofisiológica. Cognitive Brain Reserch Unit, Art. Epub. 2010;91(1):55-67.

2. Simões EL, Bramati I, Rodrigues E, Franzoi A, Moll J, Cuaresma R, et al. Expansión funcional de la representación sensoriomotriz y reorganización estructural de las conexiones callosas en amputados de miembro inferior. The Journal of Neuroscience 29 Feb. 2012.

3. Morales Osorio MA, Mejía Mejía J. Imaginería motora graduada en el síndrome de MF con dolor. Art. esp. n 808. Rev SED Esp. 2012;19.

4. Pascual L, Freitas A, Oberman C, Horveth L, Halko JC, Eldaief M. Características de la plasticidad cortical del cerebro y la dinámica de la red en todo el período de edad en la salud y la enfermedad con TMS-EEG y TMS-fMRI. Topografía cerebral 2011;24. 302-315.doi 10.1007/10548011-0196-8.

5. Noriega K, Pacheco J, Villanueva D. Neuroplasticidad (Diap 53). 2011. Disponible en: http://www.slideshare.net/ jorge_p/neuroplasticidad-1312515.

6. Feinberg TE, Farah MJ. Disorders of body perception. En: Behavioral Neurology and Neuropsichology. Nueva York: McGraw-Hill; 1997.

7. Sirigu A, Grafman J, Bressier K, Sunderland T. Multiple representation contribute to body knowledge processing. Brain 1991:629-42.

8. Kolb B, Whishaw IQ. Fundamentos de Neuropsicología Humana. Barcelona: Ed. Labor; 1986. p. 552-73.

9. Bayona Prieto J, León-Sarmiento FE, Bayona EA. Neurorehabilitación. En: Uribe CS, Arana A, Lorenzana P, editores. 7. ${ }^{\mathrm{a}}$ ed. Medellín CIB; 2009.

10. Pick A. Zhur Pathaologie des Bewubtseins von eigenen Korper-Ein Beitrag aus der Kreigsmedizin. Neurol Zentralbl 1915;34:257-65.

11. Stetter E. Zur Phaenomenologie des Phantom-gliedes. Dtsch Z Neverheilk 1950;163:141-71.

12. Martínez-Parra C, Viñuela F, Duque P. Alteraciones del esquema corporal. Cap. 13. 2011. Disponible en: http:// findpdf.net/pdf-viewer/alteraciones-del-esquema-corporal

13. Montoya P. Fundamentos neurobiológicos del dolor en el miembro fantasma. Univ. de las Islas Baleares; 2009.

14. Teddy PJ, Bittar RG. Peripheral neuromodulation for pain. J Clin Neurosci 2009.

15. Greenfield SA. The private life of the brain. London: Penguin; 2000 .

16. Casasanto D. Neuromodulation and Neural Plasticity. Disponible en: http//: serendip. brynmawr. edu/bb/ neuro98/202s98-paper2/Casasanto2.html. Consultado el 29/11/2009.

17. Librar MC, Ziemman U. Determinantes de la Inducción de la plasticidad cortical de la estimulación cerebral no invasiva en sujetos sanos. J Physiol 2010.

18. León S. FE, Rehabilitación y restauración nutricional en neurología. Salud UIS 1998.

19. Fridman EA. Plasticidad cerebral y aprendizaje en la neurorehabilitación. Arch Neural Neurocir/Neuropsiq 2004;8:40-53.

20. Pascual Leone A, Tormos-Muñoz JM. Estimulación magnética transcraneal: fundamentos y potencial de la modulación de redes neuronales específicas. Rev Neurología (Barcelona) 2008.

21. Knotkova H, Cruciani RA, Tronnier VM, Rasche D. Las opciones actuales y futuras para el tratamiento del dolor del miembro fantasma. J Res Dolor 2012;5:39-49. 